(C) 2019. This manuscript version is made available under the CC-BY-NC-ND 4.0 license http:// creativecommons.org/licenses/by-nc-nd/4.0/

\title{
Characterizing identifying codes from the spectrum of a graph or digraph *
}

\author{
C. Balbuena ${ }^{1}$, C. Dalfó ${ }^{2}$, B. Martínez-Barona ${ }^{1}$ \\ ${ }^{1}$ Departament d'Enginyeria Civil i Ambiental, Universitat Politècnica de Catalunya, \\ Barcelona, Catalonia, Spain. \\ ${ }^{2}$ Departament de Matemàtica, Universitat de Lleida, \\ Igualada (Barcelona), Catalonia.
}

e-mails:\{m.camino.balbuena,berenice.martinez\}@upc.edu, cristina.dalfo@matematica.udl.cat

\begin{abstract}
A $(1, \leq \ell)$-identifying code in digraph $D$ is a dominating subset $C$ of vertices of $D$, such that all distinct subsets of vertices of $D$ with cardinality at most $\ell$ have distinct closed in-neighborhoods within $C$. As far as we know, it is the very first time that the spectral graph theory has been applied to the identifying codes. We give a new method to obtain an upper bound on $\ell$ for digraphs. The results obtained here can also be applied to graphs.
\end{abstract}

Mathematics Subject Classifications: 05C69, 05C20, 05C50.

Keywords: Graph, digraph, identifying code, adjacency matrix, spectrum, eigenvectors.

\section{Introduction}

We consider simple digraphs (or directed graphs) without loops or multiple edges. Unless otherwise stated, we follow the textbook by Bang-Jensen and Gutin [4] for terminology

${ }^{*}$ This research is supported by MINECO under project MTM2014-60127-P, and the Catalan Research Council under project 2014SGR1147. B. Martínez-Barona has received funding from the scholarship 254379/438356 from CONACYT from Mexico.

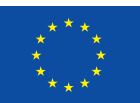

The second and third authors have also received funding from the European Union's Horizon 2020 research and innovation programme under the Marie Skłodowska-Curie grant agreement No 734922 . 
and definitions. Besides, regarding spectral graph theory, we use the notation of Godsil and Royle [8].

Let $D=(V, E)$ be a digraph with vertex set $V$ and arc set $E$. A vertex $u$ is adjacent to a vertex $v$ if $(u, v) \in E$. If there exist $\operatorname{arcs}(u, v)$ and $(v, u)$, then we say that both arcs form a digon. A digraph is symmetric if $(u, v) \in E$ implies $(v, u) \in E$. A digon is often called a symmetric arc of $D$. A digraph $D$ is said to be oriented if it has no digon. The girth $g$ of a digraph is the length of a shortest cycle. Hence, an oriented digraph has girth $g \geq 3$. Moreover, observe that every (undirected) graph $G$ with vertex set $V$ and edge set $E$ can be seen as a symmetric digraph denoted by $\stackrel{\leftrightarrow}{G}$ replacing each edge $u v \in E$ by the digon $(u, v)$ and $(v, u)$. In other words, a graph can be seen as a symmetric digraph.

The out-neighborhood of a vertex $u$ is $N^{+}(u)=\{v \in V:(u, v) \in E\}$ and the inneighborhood of $u$ is $N^{-}(u)=\{v \in V:(v, u) \in E\}$. The closed in-neighborhood of $u$ is $N^{-}[u]=\{u\} \cup N^{-}(u)$, and the closed out-neighborhood of $u$ is defined analogously. The out-degree of $u$ is $d^{+}(u)=\left|N^{+}(u)\right|$ and its in-degree $d^{-}(u)=\left|N^{-}(u)\right|$. We denote by $\delta^{+}=\delta^{+}(D)$ the minimum out-degree of the vertices in $D$, and by $\delta^{-}=\delta^{-}(D)$ the minimum in-degree. The minimum degree of $D$ is $\delta=\delta(D)=\min \left\{\delta^{+}(D), \delta^{-}(D)\right\}$. A digraph $D$ is said to be $d$-in-regular if $\left|N^{-}(v)\right|=d$ for all $v \in V$, and $d$-regular if $\left|N^{+}(v)\right|=\left|N^{-}(v)\right|=d$ for all $v \in V$.

The spectrum of the adjacency matrix $A$ of a graph $G$ or digraph $D$ is denoted by $\operatorname{sp}(A)=\left\{\lambda_{0}^{m_{0}}, \lambda_{1}^{m_{1}}, \ldots, \lambda_{d}^{m_{d}}\right\}$, where $\lambda_{i}$ are the different eigenvalues and the superscripts stand for their (algebraic) multiplicities $m_{i}=m\left(\lambda_{i}\right)$, whereas $e v(A)$ is the set of the different eigenvalues (without their multiplicities).

Given a vertex subset $U \subset V$, let $N^{-}[U]=\bigcup_{u \in U} N^{-}[u]$. For a given integer $\ell \geq 1$, a vertex subset $C \subset V$ is a $(1, \leq \ell)$-identifying code in $D$ when, for all distinct subsets $X, Y \subset V$, with $1 \leq|X|,|Y| \leq \ell$, we get

$$
N^{-}[X] \cap C \neq N^{-}[Y] \cap C .
$$

The definition of a $(1, \leq \ell)$-identifying code for graphs was introduced by Karpovsky, Chakrabarty, and Levitin [9], where the minus sign superscripts in (1) can be omitted. Thus, the definition for digraphs is a natural extension of the concept of $(1, \leq \ell)$-identifying codes in graphs. A $(1, \leq 1)$-identifying code is referred to as an identifying code.

Laihonen [10] proved the following result for graphs.

Theorem 1.1. [10] Let $k \geq 2$ be an integer.

1. If a $k$-regular graph has girth $g \geq 7$, then it admits a $(1, \leq k)$-identifying code.

2. If a $k$-regular graph has girth $g \geq 5$, then it admits a $(1, \leq k-1)$-identifying code.

Besides, Laihonen and Ranto [11] showed that if $G$ is a connected graph with at least 


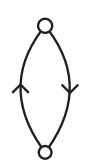

(a)

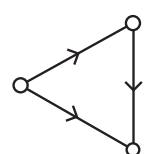

(b)

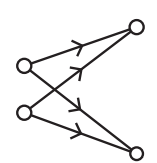

(c)

Figure 1: The subdigraphs forbidden by Lemma 1.1: (a) The digon, (b) $T T_{3}$, and $(c)$ $B T_{2,2}$.

three vertices admitting a $(1, \leq \ell)$-identifying code, then $\ell \leq \delta$, where $\delta$ is the minimum degree of $G$.

Regarding digraphs, the authors proved in [2] that every 1-in-regular digraph has a $(1, \leq 2)$-identifying code if and only if its girth is at least 5 . They also characterized the 2-in-regular digraphs having a $(1, \leq 2)$-identifying code or a $(1, \leq 3)$-identifying code. Moreover, they gave some sufficient conditions for a digraph of minimum in-degree $\delta^{-} \geq 2$ to admit a $\left(1, \leq \delta^{-}\right)$-identifying code. As a corollary of this result, they proved that a graph of minimum degree $\delta \geq 2$ and girth at least 7 admits a $(1, \leq \delta)$-identifying code.

Recall that if $D$ admits a $(1, \leq \ell)$-identifying code, then it admits a $\left(1, \leq \ell^{\prime}\right)$-identifying code for any $\ell^{\prime}<\ell$.

A digraph with adjacency matrix $A=\left(a_{u v}\right)$ has eigenvalue $\lambda$ and eigenvector $\boldsymbol{x}=\left(x_{u}\right)$ if and only if

$$
A \boldsymbol{x}=\lambda \boldsymbol{x} \quad \Leftrightarrow \quad \sum_{v \in V} a_{u v} x_{v}=\sum_{v \in N^{+}(u)} x_{v}=\lambda x_{u} \quad \text { for all } u \in V .
$$

This last equation leads to the charge displacement interpretation; for more information about it, see Fiol and Mitjana [6] or Godsil [7]. Moreover, the spectral radius of $A$ is the largest among the absolute values of its eigenvalues.

Recall also that a transitive tournament $T T_{3}$ if formed by vertices $u, v$, and $w$, and arcs $(u, v),(u, w)$, and $(v, w)$. Besides, we called bipartite tournament $B T_{2,2}$ to the digraph formed by vertices $u, v, w$ and $x$, and $\operatorname{arcs}(u, w),(u, x),(v, w)$ and $(v, x)$. See both digraphs in $1(b)$ and $1(c)$, respectively.

Our first lemma is the only non-spectral result of this paper.

Lemma 1.1. Let $D$ be a d-in-regular digraph on $n$ vertices, without any of the subdigraphs of Figure 1. If $D$ admits $a(1, \leq \ell)$-identifying code, then $\ell \in\{d, d+1\}$.

Proof. Theorem 2 (ii) in [2] states the following: Let $D$ be a twin-free digraph with minimum in-degree $\delta^{-} \geq 1$. Suppose that $D$ does not contain any subdigraph as those of Figure 1 , then $D$ admits a $\left(1, \leq \delta^{-}\right)$-identifying code. Besides, Corollary 2 in [2] states: Let $D$ be a digraph admitting a $(1, \leq \ell)$-identifying code. Then, $\ell \leq \min \left\{d^{-}(u)+1 \mid u \in\right.$ $V(D)$ and $\left.d^{+}(u) \geq 1\right\}$. 


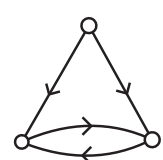

(a)

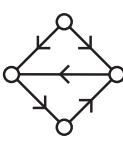

(b)

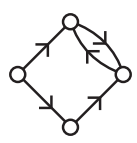

(c)

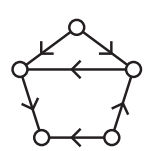

(d)

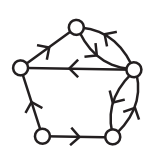

(e)

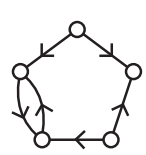

(f)

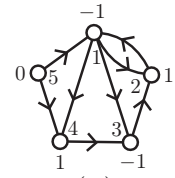

(g)

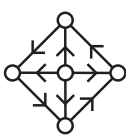

(h)

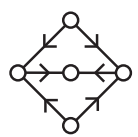

(i)

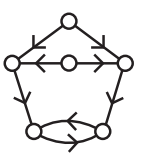

(j)

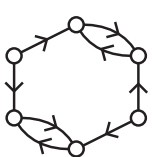

(k)

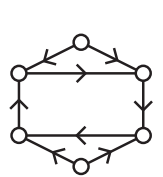

(l)

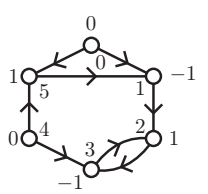

(m)

Figure 2: The forbidden subdigraphs characterizing a 2-in-regular digraph admitting a $(1, \leq 1)$-identifying code (only $(a)$ ), or a $(1, \leq 2)$-identifying code (all of them). The subdigraph $(g)$ and $(m)$ have their vertices numbered in the interior, and the entries of the eigenvector corresponding to eigenvalue -1 in the exterior.

For our $d$-in-regular digraphs, from the first result, we get that $\ell \geq d$; and, from the second one, $\ell \leq d+1$. So, $\ell \in\{d, d+1\}$.

\section{Main results}

We begin with a result that gives a sufficient (spectral) condition for a digraph to admit a $(1, \leq 1)$-identifying code.

Lemma 2.1. Let $D$ be a digraph with adjacency matrix $A$ and with a set of eigenvalues denoted by $\operatorname{ev}(A)$. If $-1 \notin \mathrm{ev}(A)$, then $D$ admits a $(1, \leq 1)$-identifying code.

Proof. We reason by contradiction. The digraph $D$ does not admit a $(1, \leq 1)$-identifying code if and only if there exists a pair of vertices $u$ and $v$ such that $N^{-}[u]=N^{-}[v]$. So, $(A+$ $I) \boldsymbol{e}_{\boldsymbol{u}}=(A+I) \boldsymbol{e}_{\boldsymbol{v}}$, where $\boldsymbol{e}_{\boldsymbol{u}}$ and $\boldsymbol{e}_{\boldsymbol{v}}$ are the unitary characteristic vectors corresponding to vertices $u$ and $v$, respectively, and $I$ is the identity matrix. Then, $(A+I) \boldsymbol{x}=\mathbf{0}$ with $\boldsymbol{x}=\boldsymbol{e}_{\boldsymbol{u}}-\boldsymbol{e}_{\boldsymbol{v}}$, whence $A \boldsymbol{x}=-\boldsymbol{x}$ and $-1 \in e v(A)$.

Remark 2.1. The converse is not true since, if $-1 \in \operatorname{ev}(A)$, this does not imply that some of its corresponding eigenvectors are of the form $\boldsymbol{e}_{\boldsymbol{i}}-\boldsymbol{e}_{\boldsymbol{j}}$. For example, the digraph in Figure $2(j)$ has -1 as an eigenvalue, but it does admit a $(1, \leq 1)$-identifying code.

In [2] the authors gave the following theorem, which is a combinatorial characterization of a 2 -in-regular digraph admitting a $(1, \leq 1)$-, $(1, \leq 2)$-, or $(1, \leq 3)$-identifying code.

Theorem 2.1 ([2]). Let $D$ be a 2-in-regular digraph. Then,

(i) $D$ admits a $(1, \leq 1)$-identifying code if and only if it does not contain any subdigraph isomorphic to Figure $2(a)$. 
(ii) $D$ admits a $(1, \leq 2)$-identifying code if and only if it does not contain any subdigraph isomorphic to one of the digraphs of Figure 2.

(iii) $D$ admits a $(1, \leq 3)$-identifying code if and only if it is oriented, $T T_{3}$-free and does not contain any subdigraph isomorphic to one of the digraphs of Figure 3.

Next, we present an algebraic-combinatorial sufficient condition for a 2-in-regular digraph to admit a $(1, \leq 2)$ - or $(1, \leq 3)$-identifying code, but first we need the following lemma.

Lemma 2.2. Let $D^{\prime}$ be a digraph with maximum in-degree $\Delta^{-}$having an eigenvalue $\lambda$ with eigenvector $\boldsymbol{x}^{\prime}=\left(x_{u}^{\prime}\right)$, such that $x_{v}^{\prime}=0$ for any vertex $v \in V\left(D^{\prime}\right)$ with $d^{-}(v)<\Delta^{-}$. Then, any $\Delta^{-}$-in-regular digraph $D$ containing $D^{\prime}$ as a subdigraph has also the eigenvalue $\lambda$.

Proof. Let $A^{\prime}=A^{\prime}\left(D^{\prime}\right)$ be the adjacency matrix of $D^{\prime}$. We know that $A^{\prime} \boldsymbol{x}^{\prime}=\lambda \boldsymbol{x}^{\prime}$. Let $A$ be the adjacency matrix of $D$ containing $D^{\prime}$ as a subdigraph, such that $a_{i j}=a_{i j}^{\prime}$ for all $i, j \in V\left(D^{\prime}\right)$. Now let us show that $\lambda$ also is an eigenvalue of $A$. To see this, it is enough to check that vector $\boldsymbol{x}$, obtained from $\boldsymbol{x}^{\prime}$ by adding zeros to the entries of $D$ corresponding to the vertices that are not in $D^{\prime}$, is an eigenvector of $A$ with eigenvalue $\lambda$. Indeed, from (2), for all $u \in V$ we get

$$
\sum_{v \in N^{+}(u)} x_{v}=\sum_{\substack{v \in N^{+}(u) \\ v \in V^{\prime}}} x_{v}+\sum_{\substack{v \in N^{+}(u) \\ v \notin V^{\prime}}} x_{v}=\sum_{\substack{v \in N^{+}(u) \\ v \in V^{\prime}}} x_{v}
$$

because by the construction of vector $\boldsymbol{x}$, the sum when $v \notin V^{\prime}$ is zero. Then,

- If $u \in V^{\prime}$, then (3) gives

$$
\sum_{v \in N^{+}(u)} x_{v}=\lambda x_{u}
$$

- If $u \notin V^{\prime}$, then (3) provides

$$
\sum_{v \in N^{+}(u)} x_{v}=0=\lambda x_{u}
$$

because, again by the construction of $\boldsymbol{x}$, both $\sum_{\substack{v \in N^{+}(u) \\ v \in V^{\prime}}} x_{v}$ and $x_{u}$ are zero.

Now we give an algebraic-combinatorial sufficient condition for a 2-in-regular digraph to admit a $(1, \leq 2)$-, or $(1, \leq 3)$-identifying code. By appealing to the eigenvalues of the digraphs we can reduce the number of forbidden subdigraphs considered in Theorem 2.1.

Theorem 2.2. Let $D$ be a 2-in-regular digraph with adjacency matrix A. 


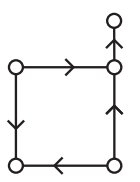

(a)

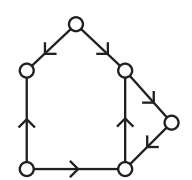

(i)

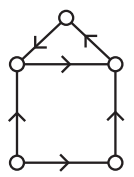

(b)

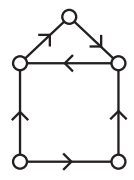

(c)

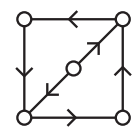

(d)

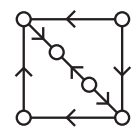

(e)

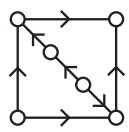

$(f)$

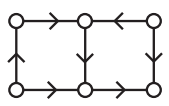

$(g)$

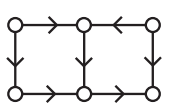

(h)

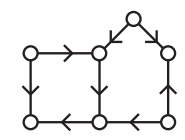

(j)

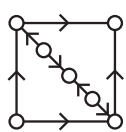

(k)

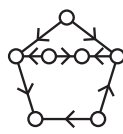

$(\ell)$

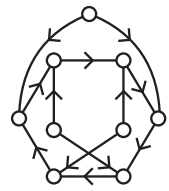

(m)

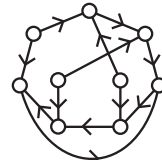

(n)

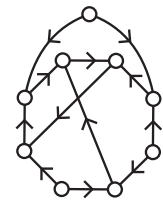

(o)

Figure 3: The forbidden subdigraphs characterizing a $T T_{3}$-free, 2-in-regular and oriented digraph admitting a $(1, \leq 3)$-identifying code.

(i) If $-1 \notin \mathrm{ev}(A)$ and $D$ does not contain any subdigraph isomorphic to $(b),(c),(d)$, $(f)$ and $(i)$ of Figure 2, then $D$ admits a $(1, \leq 2)$-identifying code.

(ii) If $-1,0 \notin \mathrm{ev}(A)$ and $D$ does not contain any subdigraph isomorphic to $(b)-(\ell)$ of Figure 3, then $D$ admits a $(1, \leq 3)$-identifying code.

Proof. To prove $(i)$, by Theorem 2.1(ii), we know that if a 2-in-regular digraph $D$ does not contain any of the subdigraphs of Figure 2 , then $D$ admits a $(1, \leq 2)$-identifying code. The subdigraphs $(a),(e),(g),(h)$ and $(j)-(m)$ satisfy Lemma 2.2 for $\lambda=-1$. Then, we only need to forbid the subdigraphs $(b),(c),(d),(f)$ and $(i)$ to get the result. By way of example, in Figure 2, digraphs $(g)$ and $(m)$ have their vertices numbered in the interior, and the entries of the eigenvector corresponding to eigenvalue -1 in the exterior, that is, their charges (see Eq. (2)). In both digraphs the vertices of in-degree less than $\Delta^{-}=2$ have charge 0 , so satisfy Lemma 2.2 . To prove $(i i)$, reasoning similarly, we get that the subdigraph $(a)$ of Figure 3 satisfies Lemma 2.2 for $\lambda=0$, and subdigraphs $(m)-(o)$ satisfy Lemma 2.2 for $\lambda=-1$, so we only need to forbid the rest of the subdigraphs in this figure.

We provide some necessary notation introduced by Powers [12] and referenced in the book by Cvetković, Rowlinson and Simić [5]. Let $\boldsymbol{x}=\left(x_{i}\right)$ be an eigenvector associated with an eigenvalue $\lambda$ different from the spectral radius, and let $\boldsymbol{z}$ be an eigenvector associated with the spectral radius. We denote by $\mathcal{P}(\boldsymbol{x})$ and $\mathcal{N}(\boldsymbol{x})$ the set of its positive and negative entries, respectively. That is, $\mathcal{P}(\boldsymbol{x})=\left\{i: x_{i}>0\right\}$ and $\mathcal{N}(\boldsymbol{x})=\left\{i: x_{i}<0\right\}$. Since $\boldsymbol{x}$ and $\boldsymbol{z}$ are orthogonal, $\mathcal{P}(\boldsymbol{x})$ and $\mathcal{N}(\boldsymbol{x})$ are nonempty, because all the entries of $\boldsymbol{z}$ are positive.

Let us show the meaning of the sign of a real eigenvalue on the sets of in-neighborhoods of vertices.

Proposition 2.1. Let $D=(V, E)$ be a digraph with adjacency matrix $A$ having some real eigenvalue, say $\lambda \in \operatorname{ev}(A)$, different from the spectral radius. Let $\boldsymbol{x}=\left(x_{u}\right)_{u \in V}$ be an 
eigenvector of $A$ associated with $\lambda$ such that $X=\mathcal{P}(\boldsymbol{x})$ and $Y=\mathcal{N}(\boldsymbol{x})$. Then, depending on the sign of $\lambda$, the following holds:

(a) If $\lambda<0$, then $X \cup N^{-}(X)=Y \cup N^{-}(Y) \quad\left(\Leftrightarrow N^{-}[X]=N^{-}[Y]\right)$.

(b) If $\lambda>0$, then $X \cup N^{-}(Y)=Y \cup N^{-}(X)$.

(c) If $\lambda=0$, then $N^{-}(X)=N^{-}(Y)$.

Proof. Let $\boldsymbol{\Phi}_{N^{-}(i)}=A \mathbf{e}_{i}$ denote the characteristic vector of the open in-neighbourhood of vertex $i$, where $\mathbf{e}_{i}$ is the unitary characteristic vector of $i$. Let $\boldsymbol{x}_{+}$be the vector obtained from a vector $\boldsymbol{x}$ by changing all its negative components to zero. Similarly, $\boldsymbol{x}_{-}$is obtained from $\boldsymbol{x}$ by changing all its positive components to zero. Then, $\boldsymbol{x}=\boldsymbol{x}_{+}+\boldsymbol{x}_{-}$. Since $\boldsymbol{x}$ is a $\lambda$-eigenvector of $A$, we get $A \boldsymbol{x}=\lambda \boldsymbol{x}$ or $A \boldsymbol{x}-\lambda \boldsymbol{x}=\mathbf{0}$. Now we distinguish the possible cases according to the sign of $\lambda$ :

(a) and (b): If $\lambda<0$ or $\lambda>0$, we have

$$
\sum_{i \in X} x_{i} \boldsymbol{\Phi}_{N^{-}(i)}-\lambda \boldsymbol{x}_{+}+\sum_{j \in Y} x_{j} \boldsymbol{\Phi}_{N^{-}(j)}-\lambda \boldsymbol{x}_{-}=\mathbf{0} .
$$

For the case $\lambda<0, \sum_{i \in X} x_{i} \boldsymbol{\Phi}_{N^{-}(i)}$ and $-\lambda \boldsymbol{x}_{+}$are positive, whereas $\sum_{j \in Y} x_{j} \boldsymbol{\Phi}_{N^{-}(j)}$ and $-\lambda \boldsymbol{x}_{-}$are negative. Thus, for the global sum to be $\mathbf{0}$, we must have $N^{-}[X]=N^{-}[Y]$. For the case $\lambda>0, \sum_{i \in X} x_{i} \boldsymbol{\Phi}_{N^{-}(i)}$ and $-\lambda \boldsymbol{x}_{-}$are positive, whereas $-\lambda \boldsymbol{x}_{+}$and $\sum_{j \in Y} x_{j} \boldsymbol{\Phi}_{N^{-}(j)}$ are negative. Then, the global sum is $\mathbf{0}$ if $X \cup N^{-}(Y)=Y \cup N^{-}(X)$.

(c) Finally, if $\lambda=0$, the vector equality

$$
\sum_{i \in X} x_{i} \boldsymbol{\Phi}_{N^{-}(i)}+\sum_{j \in Y} x_{j} \boldsymbol{\Phi}_{N^{-}(j)}=\mathbf{0}
$$

yields the result.

The same result holds for graphs by changing $N^{-}(X)$ and $N^{-}(Y)$ by $N(X)$ and $N(Y)$, respectively. Moreover, a similar result concerning out-neighborhoods (instead of in-neighborhoods) can be obtained by applying Proposition 2.1 to the converse digraph of $D$ or, equivalently, considering the left (instead of right) eigenvectors of $D$. The next result gives an upper bound for $\ell$ in a digraph $D$ having a $(1, \leq \ell)$-identifying code.

Corollary 2.1. Let $D$ be a digraph admitting a $(1, \leq \ell)$-identifying code. Let $A$ be its adjacency matrix having at least one negative eigenvalue $-\lambda$ (with $\lambda>0$ ) with $\boldsymbol{x}=$ $\left(x_{1}, \ldots, x_{n}\right)$ any associated eigenvector. Then $\ell<\min _{\boldsymbol{x}} \max \{|\mathcal{P}(\boldsymbol{x})|,|\mathcal{N}(\boldsymbol{x})|\}$.

Example 2.1. Consider the digraph of Figure 2 $(m)$. Its spectrum is $\left\{0^{4}, 1^{1},-1^{1}\right\}$. An eigenvector corresponding to the eigenvalue -1 is $(0,-1,1,-1,0,1)$. The positions of the positive entries of this eigenvector give us vertex subset $X=\{2,5\}$, and the positions of the 
negatives entries give $Y=\{1,3\}$. We can check that $N^{-}[X]=N^{-}[Y]=\{0,1,2,3,4,5\}$. Then, this digraph does not admit a $(1, \leq 2)$-identifying code.

An eigenvector corresponding to the eigenvalue -1

To give a general result not depending on some specific eigenvector, but only on the multiplicity of the corresponding eigenvalue, we give the following lemma. More precisely, the next result shows that, given a real eigenvalue with geometric multiplicity $m$, some of its eigenvectors can be chosen with at least $m-1$ zero entries.

Theorem 2.3. Let $D$ be a digraph on $n$ vertices with adjacency matrix $A$, and let $\lambda$ be a real eigenvalue of $A$ with geometric multiplicity $m$. For any given index set $I \subset$ $\{1,2, \ldots, n\}$ with $|I|=m-1$, there exists an eigenvector $\boldsymbol{x}$ with eigenvalue $\lambda$ and entries $x_{i}=0$ for every $i \in I$.

Proof. Every eigenvector $\boldsymbol{x}=\left(x_{1}, \ldots, x_{n}\right)$ associated with the eigenvalue $\lambda$ is a solution of the homogeneous system $(A-\lambda I) \boldsymbol{x}=\mathbf{0}$. Since the geometric multiplicity of $\lambda$ is $m$, it follows that the rank of $A-\lambda I$ is $n-m>0$. Hence, there is a triangular matrix $B$ having null the last $m$ rows, and diagonal entries $b_{i}^{i}=1$ or $b_{i}^{i}=0$, for $i=1, \ldots, n-m$, such that $B \cdot \boldsymbol{x}=\mathbf{0}$. Thus, each of the first $n-m$ components of $\boldsymbol{x}$ can be expressed as a linear combination of the last $m$ ones, that is,

$$
x_{i}=\sum_{j=n-m+1}^{n} \alpha_{j}^{i} x_{j}, \quad i=1, \ldots, n-m .
$$

This implies that

$$
\boldsymbol{x}=\sum_{j=n-m+1}^{n}\left(\alpha_{j}^{1} x_{j}, \ldots, \alpha_{j}^{n-m} x_{j}, x_{n-m+1}, \ldots, x_{m}\right),
$$

yielding that the set of $m$ vectors

$$
\begin{gathered}
\left(\alpha_{n-m+1}^{1}, \ldots, \alpha_{n-m+1}^{n-m}, 1,0, \ldots, 0\right), \\
\left(\alpha_{n-m+2}^{1}, \ldots, \alpha_{n-m+2}^{n-m}, 0,1,0, \ldots, 0\right), \\
\vdots \\
\left(\alpha_{n}^{1}, \ldots, \alpha_{n}^{n-m}, 0, \ldots, 0,1\right) .
\end{gathered}
$$

is a base of eigenvectors associated with $\lambda$, and clearly, all of them have at least $m-1$ zero entries.

With the following remark, we state that our results can be applied to graphs. 


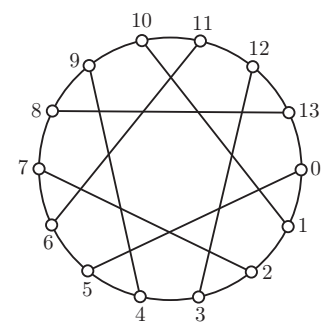

Figure 4: The Heawood graph.

Remark 2.2. Corollary 2.1 can also be applied to graphs, which always have real eigenvalues.

Let us see an example on a graph.

Example 2.2. Consider the Heawood graph (see Figure 4). Its spectrum is $\left\{3^{1}, \sqrt{2}^{6},-\sqrt{2}^{6}\right.$, $\left.-3^{1}\right\}$. The eigenvector corresponding to -3 and the 6 eigenvectors of $-\sqrt{2}$ are, respectively,

$$
\begin{aligned}
& (-1,1,-1,1,-1,1,-1,1,-1,1,-1,1,-1,1) \\
& (-1,0,1,0,-1, \sqrt{2}, 0,-\sqrt{2}, 1,0,0,0,0,0), \\
& (-1,0,1,-\sqrt{2}, 0, \sqrt{2},-1,0,0,0,0,0,1,0), \\
& (0,-1, \sqrt{2},-1,0,1,-\sqrt{2}, 0,0,0,0,1,0,0), \\
& (0,-1, \sqrt{2}, 0,-\sqrt{2}, 1,0,-1,0,1,0,0,0,0) \\
& (0,-\sqrt{2}, 1,0,-1, \sqrt{2},-1,0,0,0,1,0,0,0) \\
& (-\sqrt{2}, 0, \sqrt{2},-1,0,1,0,-1,0,0,0,0,0,1) .
\end{aligned}
$$

The last one is one of the eigenvectors whose entries have the maximum number of zeros and with the minimum difference between the number of positive and negative entries. The positions of the positive entries of this eigenvector give us the set of vertices $X=\{2,5,13\}$. The positions of the negatives entries give the set $Y=\{0,3,7\}$. We can check that $N^{-}[X]=N^{-}[Y]=\{0,1,2,3,4,5,6,7,8,12,13\}$. Then, the Heawood graph does not admit $a(1, \leq 3)$-identifying code. By Theorem 1.1 we know that the Heawood graph, which is 3-regular and it has girth 6 , admits a $(1, \leq 2)$-identifying code.

\section{References}

[1] G. Araujo-Pardo, C. Balbuena, L. Montejano, and J. C. Valenzuela, Partial linear spaces and identifying codes, European J. Combin. 32 (2011) 344-351.

[2] C. Balbuena, C. Dalfó, and B. Martínez, Sufficient conditions for a digraph to admit a $(1, \leq \ell)$-identifying code, submitted, 2017. 
[3] C. Balbuena and J. Salas, On the order of graphs with a given girth pair, Discrete Math. 321 (2014) 68-75.

[4] J. Bang-Jensen and G. Gutin, Digraphs: Theory, Algorithms and Applications, Springer-Verlag, London, 2007.

[5] D. Cvetković, P. Rowlinson, and S. K. Simić, An introduction to the theory of graph spectra, London Mathematical Society Student Texts, 75, Cambridge: Cambridge University Press, 2010.

[6] M. A. Fiol and M. Mitjana, The spectra of some families of digraphs, Linear Algebra Appl. 423 (2007) 109-118.

[7] C. D. Godsil, Algebraic Combinatorics, Chapman and Hall, New York, 1993.

[8] C. Godsil and G. Royle, Algebraic Graph Theory, Springer-Verlag, New York, 2001.

[9] M. Karpovsky, K. Chakrabarty, and L. Levitin, On a new class of codes for identifying vertices in graphs, IEEE Trans. Inform. Theory 44 (1998) 599-611.

[10] T. Laihonen, On cages admitting identifying codes, European J. Combin. 29 (2008) 737-741.

[11] T. Laihonen and S. Ranto, Codes identifying sets of vertices, Lect. Notes Comput. Sci. 2227 (2001) $82-91$.

[12] D. L. Powers, Graph partitioning by eigenvectors, Linear Algebra Appl. 101 (1988) 121-133. 